\title{
Interacción colaborativa en aplicación móvil: una propuesta de enseñanza híbrida en clases de español
}

\section{Collaborative interaction in mobile application: a hybrid-teaching proposal in Spanish classes}

\author{
Rosana Beatriz Garrasini Sellanes ${ }^{1}$ \\ Iris Oliveira de Carvalho ${ }^{2}$
}

\begin{abstract}
Resumen
Este artículo presenta una investigación cualitativa, también una observación participante y un estudio de caso, hecha con estudiantes de la Enseñanza Fundamental (EF) de una escuela pública Federal, de la región norte de la ciudad de Goiânia, en el Estado de Goiás, Brasil. En este estudio se propuso una actividad de interacción colaborativa en aplicación de mensajería móvil en las clases de Español Lengua Extranjera (ELE). La propuesta se justifica pues las clases de Lengua Extranjera (LE) ocupan muy poco el horario de clases con un máximo de una hora y media por semana. Este tipo de actividad que mezcla la enseñanza en clase y en contexto digital se encuadra en la perspectiva de la enseñanza híbrida y tiene como objetivo principal fomentar la autonomía y la colaboración entre los estudiantes en otros ambientes de enseñanza diferentes de la clase. Los supuestos teóricos utilizados en este estudio se basan en la teoría sociocultural de Vygotsky para explicar la interacción colaborativa y el andamiaje entre los alumnos y/o la profesora. También la teoría de la enseñanza en contexto híbrido y la utilización de aplicación móvil de mensajería como herramienta pedagógica. Se percibe en algunos estudios que la utilización de aplicaciones móviles es una tendencia entre los profesores de diversos niveles y que ha presentado muy buenos resultados para la enseñanza y aprendizaje de lenguas.
\end{abstract}

Palabras clave: Interacción colaborativa. Educación híbrida. Español lengua extranjera.

\begin{abstract}
This article presents a qualitative investigation, also a participant observation and a case study, made with students of the Fundamental Education of a federal public school, in the northern region of the city of Goiânia, in the State of Goiás, Brazil. In this research, a collaborative interaction activity was proposed in the mobile messaging application in the Spanish foreign language classes. The proposal is justified because the Foreign Language (LE) classes occupy very little the class schedule with a maximum of one hour and a half per week. This type of activity that mixes teaching in class and in a digital context is framed in the perspective of hybrid teaching and its main objective is to promote autonomy and collaboration among students in other teaching environments different from the classroom. The theoretical assumptions used in this study are based on Vygotsky's sociocultural theory to explain collaborative interaction and scaffolding between students and / or the teacher. In addition, the theory of teaching in blended context and the use of mobile messaging application as a pedagogical tool. It is perceived in some studies that the use of mobile applications is a trend among teachers of various levels and has presented great results for teaching and learning languages.
\end{abstract}

Keywords: Collaborative interaction. Blended education. Spanish foreign language.

\footnotetext{
${ }_{1}^{1}$ Mestre em Ensino na Educação Básica. CEPAE/UFG (Centro de Ensino e Pesquisa Aplicada à Educação da Universidade Federal de Goiás), Goiânia, Goiás, Brasil. Orcid: https://orcid.org/0000-0003-2050-9755 E-mail: rosanagarrasini@ufg.br

2 Doutoranda e Mestre em Letras e Linguística. CEPAE/UFG (Centro de Ensino e Pesquisa Aplicada à Educação da Universidade Federal de Goiás), Goiânia, Goiás, Brasil. Orcid: https://orcid.org/0000-001-8549-5644 E-mail: irisoc@ufg.br
} 


\section{Introducción}

Los teléfonos móviles dejaron de ser meros artefactos de comunicación a la distancia para convertirse en herramientas de información, registro de imágenes y mediadores en el proceso de enseñanza y aprendizaje. Los recursos que poseen el móvil y la aceptación de la tecnología por las generaciones más jóvenes son el elemento clave para la difusión de su uso en la educación. Así los modelos de aprendizaje basados en dispositivos móviles y sus aplicaciones se caracterizan como una posibilidad de e-Learning o Mobile Learning (Aprendizaje Móvil) y los que utilizan el modelo presencial y el virtual son conocidos más específicamente como enseñanza híbrida.

La enseñanza híbrida es una modalidad de educación que trae lo mejor de ambos mundos: el online y el offline. Ese modelo de enseñanza, en términos generales, es lo que conecta estos modelos de aprendizaje: el presencial y el online. En otras palabras, en el presencial parte del proceso ocurre en el aula, donde los estudiantes interactúan entre sí mediante el intercambio de experiencias. En el online, utilizan medios digitales, como el móvil, para que el alumno tenga más autonomía al aprender. En este orden de ideas, ambos métodos son complementarios, porque proporcionan experiencias diferentes en la forma de aprendizaje.

Otro proceso llevado a cabo en ese tipo de herramienta es la interacción colaborativa que puede ser observada en el intercambio de ayudas entre los alumnos y también con el profesor. Esa perspectiva de interacción basada en la teoría sociocultural de Vygotsky y traspuesta a la enseñanza de lenguas trae el supuesto del andamiaje en algunas actividades propuestas en el aplicativo. La colaboración que ocurre entre los estudiantes para realizar determinada actividad y la ayuda al que tiene dudas, sea en el idioma o en cómo realizar la actividad, presupone el proceso de aprendizaje en la Zona de Desarrollo Proximal (ZDP) que proporciona el andamiaje del profesor o de un alumno más proficiente a otro alumno con más dificultades.

La justificación para esta investigación surgió de la práctica pedagógica de las investigadoras, que son profesoras de Español como Lengua Extranjera (ELE) en una escuela pública federal. Una coordina y la otra participa de un Proyecto de investigación denominado "Actividades colaborativas de aprendizaje en contexto híbrido: la enseñanza de lengua española con la utilización del móvil" que presenta propuestas de actividades para desarrollar el español con los alumnos de la Educación Básica a través de la aplicación móvil de mensajería.

También la necesidad de ampliar el tiempo de contacto de los estudiantes con la Lengua Extranjera (LE) y tornarlos más autónomos para aprenderla ya que en la enseñanza pública, las clases de LE son restrictas a una o dos veces a la semana, lo que significa apenas cuarenta y cinco a cincuenta 
minutos o una hora y media de contacto a la semana con la lengua meta. En la escuela investigada los estudiantes tienen dos clases de cuarenta y cinco minutos a la semana en la disciplina Español. Este tiempo de contacto es irrelevante para el desarrollo de las cuatro destrezas lingüísticas (hablar, escribir, leer y escuchar) en la lengua.

De esta manera, se propuso hacer una investigación cualitativa, también una investigación participativa y un estudio de caso con los alumnos de la Enseñanza Fundamental (EF), de una escuela pública Federal de Goiânia, Goiás-Brasil, más precisamente con el $8^{\circ}$ año, durante el segundo semestre de 2019, antes de la emergencia sanitaria impuesta por la pandemia del Coronavirus. Para ello, los estudiantes prepararon trabajos en grupo con la utilización de la aplicación móvil de mensajería WhatsApp para las negociaciones e interacciones en el desarrollo de la propuesta. El objetivo fue observar la interacción colaborativa y si hubo o no la percepción de la ZDP durante las interacciones en la aplicación. Específicamente, si les pareció efectivo, en la visión de los alumnos, el uso de la aplicación como recurso para el aprendizaje de LE. También para atestiguar si la enseñanza híbrida puede contribuir para el proceso de enseñanza y aprendizaje de LE ampliando los espacios de aprendizaje y aumentando el tiempo de exposición a la lengua meta en la escuela pública. Para la selección de los datos fueron observadas las interacciones colaborativas en la aplicación móvil, también las respuestas de los estudiantes al cuestionario sobre el proceso y algunas consideraciones durante una entrevista colectiva hecha en el aula después de la presentación de los trabajos.

Para ese estudio nos basamos en la teoría sociocultural de Vygotsky al hablar de la producción del conocimiento en la interacción colaborativa y sobre el andamiaje como supuesto teórico de la ZDP (HALL, 2001; FIGUEIREDO, 2001, 2018, 2019; VYGOTSKY, 2000). Además utilizamos también la teoría de la enseñanza híbrida (MORAN, 2015); (BASICH; TANZI NETO y TREVISANI, 2015; HORN Y STAKER, 2015), aprendizaje en ambiente virtual y el uso de la aplicación móvil de mensajería como herramienta para la enseñanza y aprendizaje de lenguas (CREMADES; MAQUEDA CUENCA y ONIEVA, 2016; PADRÓN, 2013; SOUZA, 2015).

Así este artículo está organizado en un aporte teórico que presenta algunos supuestos sobre la interacción colaborativa en clases de idiomas basada en la teoría sociocultural, también en el abordaje metodológico de la enseñanza híbrida y en el uso de la aplicación móvil de mensajería como herramienta pedagógica en clases de lenguas. Enseguida, la presentación y análisis de los datos, las consideraciones sobre la investigación y las referencias utilizadas.

Ahora empezamos con la base teórica que utilizamos en la investigación. 


\section{Interacción colaborativa}

Los principios del aprendizaje colaborativo están basados en los supuestos teóricos de Vygotsky y sus colaboradores. Según Vygotsky (2000), cuando hablamos sobre el desarrollo de un aprendiz, estamos hablando del conocimiento adquirido, es decir, de la capacidad para realizar ciertas tareas sin la ayuda de otra persona. Para Vygotsky (2000), esta capacidad de rendimiento independiente se llama Nivel o Zona de Desarrollo Real (ZDR), que se refiere a las etapas de desarrollo cognitivo ya logradas y es el resultado de procesos de desarrollo ya completados. Por lo tanto, no es posible medir el desarrollo de un individuo basado en el conocimiento ya adquirido, este análisis no puede hacerse retrospectivamente, sino prospectivamente. Para ello, debemos analizar la capacidad del individuo para realizar nuevas tareas, los procesos de desarrollo en formación.

La diferencia entre el nivel de tareas que se pueden realizar de forma independiente y las tareas que se pueden realizar con la ayuda de otros revela el desarrollo potencial del individuo, lo que se ha llamado la Zona de Desarrollo Próximo (ZDP), es decir, el desarrollo próximo se refiere a las habilidades no adquiridas pero que pueden demostrarse y/o adquirirse a través de la cooperación de alguien más experimentado. Según Vygotsky (2000), por medio de la ZDP es posible medir no solo el proceso de desarrollo hasta el momento y los procesos de maduración ya producidos, sino también los procesos que todavía están ocurriendo y que ahora solo están madurando y desarrollándose. En este sentido, es por la interacción que se crea la ZDP, que, para Vygotsky (2000, p. 112), se puede definir como

la brecha entre el desarrollo real, que generalmente se determina a través de la resolución independiente de problemas, y el desarrollo potencial, determinado a través de la resolución de problemas bajo la guía de un adulto o en colaboración con pares más capaces. [Traducción nuestra]

Durante la interacción con otros, el niño puede poner en marcha varios procesos de desarrollo que serían imposibles sin ayuda. Estos procesos se internalizan y se convierten en parte de las adquisiciones de su desarrollo individual, según Rego (2001). Para Vygotsky (2000, p. 113) la "ZDP hoy será el nivel de desarrollo real mañana".

Por lo tanto, de acuerdo con Carvalho (2003), su progreso completo como ser humano depende de su interacción con otros individuos en un grupo social particular, lo que proporciona aprendizaje histórico, cultural y socialmente organizado y crecimiento cognitivo. Influenciado por los principios marxistas Vygotsky argumenta que las actividades psicológicas presentadas por el individuo 
se originaron en sus relaciones sociales con el entorno externo. En este sentido, el ser humano no es solo el producto de un contexto social, histórico y cultural, sino un agente en la creación de este contexto.

Según Hall (2001) en las escuelas la interacción entre profesor y alumnos en clase es uno de los principales medios en que ocurre el aprendizaje. En las clases de idiomas la interacción entre los estudiantes y con el profesor posee un papel significativo, pues en los contextos comunicativos en los cuales el aprendiente participa juntamente con el uso del lenguaje se desarrollan las estructuras mentales universales que ya existen de la lengua materna y que influyen para la formación del aprendizaje de las estructuras de la segunda lengua. Así la actividad comunicativa de interacción que se realiza en LE es extremadamente similar a la que se produce en lengua materna para los diversos contextos comunicativos. Se percibe que la interacción y la colaboración entre los sujetos actuantes en clase proporcionan el desarrollo del aprendizaje.

Figueiredo (2019, p. 64) afirma que "el aprendizaje colaborativo enfatiza el papel de la interacción y la colaboración en el trabajo entre pares o en grupos de estudiantes para involucrarlos en la construcción del conocimiento". Por su lado, la interacción en clase de lenguas agrega el conjunto de oportunidades creadas para que los aprendientes se comuniquen entre sí y con el profesor en la lengua meta (FIGUEIREDO, 2018).

El término andamiaje fue utilizado por primera vez por Wood, Bruner y Ross (1976) para designar la asistencia brindada por adultos o niños más experimentados para realizar una tarea. Su traducción más cercana sería "estructura de apoyo", según Figueiredo (2001). Por lo tanto, la mediación entre el niño y el mundo, proporcionada por el adulto o por los niños más experimentados, puede entenderse como un andamiaje, es decir, durante el desarrollo psíquico humano, las actividades más complejas siempre están mediadas por alguien más experimentado, que crea un andamio, o una estructura de apoyo, para lograr sus objetivos. Por este medio, los miembros inmaduros de la sociedad se desarrollan psicológicamente y gradualmente se hacen cargo del funcionamiento de la cultura y se adaptan a las actividades culturales que deben realizarse, convirtiéndose en actividades voluntarias e independientes. Es por medio del andamiaje que el niño pasaría de ZDP a ZDR, o de un proceso interpsíquico a un proceso intrapsíquico.

De esta manera, acciones didácticas que favorezcan la interacción, la colaboración y el andamiaje entre los alumnos y entre estos y el profesor son actividades que pueden desarrollar conocimientos e internalización del aprendizaje. Así se puede lograr éxito con propuestas que involucren estas características, y por supuesto la motivación para la práctica de la LE con el uso de las tecnologías móviles en un aprendizaje híbrido.

\subsection{Enseñanza híbrida}


Moran (2015, p. 27) afirma que "híbrido significa mixto, mezclado, blended" y la educación siempre ha combinado los espacios, las actividades, los tiempos, las personas, las metodologías. Hoy día, a causa de la movilidad y conectividad se puede enseñar y aprender de distintas formas, a todo momento y en múltiples espacios. Si pensamos, la enseñanza y el aprendizaje siempre fueron híbridos, pues podemos aprender solos, con los colegas, en casa con la familia, cuando estudiamos, cuando nos divertimos, es decir de manera intencional, en procesos organizados y ambientes formales o en contextos informales, espontáneos, con éxito o fracaso.

Así la enseñanza híbrida, según Basich; Tanzi Neto; Trevisani (2015, p. 52), "es una combinación metodológica que impacta la acción del profesor en situaciones de enseñanza y la acción del estudiante en situaciones de aprendizaje". Dentro de la perspectiva de educación híbrida el medio online da autonomía y flexibilidad al estudiante, además de permitirle que aprenda solo o en interacción con otros y explote sus capacidades por medio de la colaboración siempre que sea posible fuera del aula.

El medio presencial, por otro lado, permite un intercambio de conocimiento más personal, una comunicación en tiempo real y una interacción cara a cara con los colegas y el profesor. Es decir, cada proceso, online o presencial, es complementario al otro, aumentando el tiempo de exposición al contenido y por supuesto la capacidad de absorber el conocimiento, sea en la clase, en el ordenador o por medio de aplicación móvil.

Para Horn y Staker (2015), la educación híbrida bien implementada forma la base de un sistema de aprendizaje centrado en el alumno. El alumno desarrolla la capacidad de conducir su aprendizaje y puede convertirse en un alumno eterno, lo cual es importante hoy en día cuando el conocimiento y las habilidades se vuelven obsoletos rápidamente.

\subsection{Aplicación móvil de mensajería en la enseñanza de lenguas}

Padrón (2013) afirma que el WhatsApp hoy en día es la aplicación para móviles más usual entre las personas, por la facilidad de comunicación en tiempo real, por compartir fotos, músicas y videos gratuitamente. Aun así se puede agregarla en el aula para la comunicación (escrita y auditiva) entre los alumnos y el profesor para sacar dudas del contenido, como recordatorio de tareas, fechas para entrega de tareas etc. sin necesidad de regulación del profesor o de horarios fijos para su utilización. El término WhatsApp, según la autora, "es un juego de palabras que viene de "what's up", una frase coloquial inglesa que significa “¿Qué pasa?, y "App”, la abreviatura también inglesa para “aplicaciones” (Padrón, 2013, p. 
129). La empresa WhatsApp Inc, con sede en Santa Clara-California, fue fundada en 2009 por Brian Acton y Jan Koum, dos veteranos de Yahoo.

Utilizar estas aplicaciones en el ámbito educativo se conoce como Mobile Learning o Aprendizaje Móvil, que es una de las posibilidades de e-Learning, es decir, el aprovechamiento de tecnologías móviles en el proceso de enseñanza y aprendizaje, en distintos contextos (virtual y físico).

Según Souza (2015), el aprendizaje móvil ya es bastante explotado en la enseñanza de LE, ya que existen muchas aplicaciones disponibles que fueron elaboradas para la enseñanza o práctica de las lenguas. La mayoría de las aplicaciones son destinadas a las prácticas gramaticales con ejercicios; aumento del vocabulario en la lengua meta; podcasts para la práctica de la comprensión oral, que son feeds de audio o textos para escuchar; diccionarios; entrenar el habla y la pronunciación etc. Algunas con juegos educacionales desarrollados por empresas nacionales e internacionales, pagadas o gratis. De esta manera se puede practicar la lengua en actividades extra clase en cualquier local que haya conexión de internet.

Sin embargo, la utilización de las aplicaciones exige una acción comprometida del profesor, que es el mediador del proceso y sobremanera del alumno, ya que la mayor parte de la generación digital no posee autonomía para buscar la ampliación de sus capacidades de aprendizaje cuando el objetivo está impregnado del sentimiento de deber escolar.

Así, el profesor tiene que elegir actividades que puedan ser desarrolladas en la aplicación y que sean motivadoras y tal vez desprendidas de ese valor laboral que se traduce en una tarea escolar. A pesar de los objetivos bien definidos, se debe organizar la actividad de una manera que se torne placentera y autónoma dejando libres algunos aspectos que pueden hacerse como demonstraciones de elección propia del aprendiente o de libre forma de presentación o de ejecución.

Cremades; Maqueda Cuenca y Onieva (2016) afirman que, antes del surgimiento de la aplicación móvil WhatsApp, Plester y Wood (2009) realizaron tres estudios con alumnos ingleses de 10 a 12 años sobre el textismo o el lenguaje usado en los SMS (Short Message Service). En los estudios investigaron su nivel de conocimiento sobre las características más comunes y frecuentes en el lenguaje usado en ese servicio de mensajes cortos. Los participantes tenían que traducir el textismo al lenguaje estándar y viceversa. Además, escribir mensajes para determinadas situaciones utilizando el lenguaje textista. Las tres investigaciones de Pester y Woods demostraron que, diferentemente de los que pensaban que la escritura abreviada disminuía la capacidad lingüística de los estudiantes, se trata de un uso creativo que, si trabajado, puede potenciar las destrezas comunicativas.

Similarmente, afirman los autores, se produjo la investigación de Alsaleem (2013) sobre el uso del WhatsApp como recurso para aumentar el rendimiento de sus estudiantes universitarias de inglés 
como segunda lengua (L2) en Arabia Saudí. El interés de Alsaleem era verificar si por medio de diarios hechos en la aplicación sería posible mejorar la escritura en inglés, ya que escribir en la aplicación llamaría la atención de los jóvenes de entonces. Los interlocutores eran profesores y los intercambios de mensajes duraron 6 semanas. La rúbrica de evaluación fueron los aspectos de la escritura: vocabulario, el tono y la adecuación del discurso. Los resultados demostraron que las estudiantes que usaban el WhatsApp en inglés tuvieron un adelanto notable del vocabulario y no tan claramente en el tono. De esta manera el uso del diario electrónico se convirtió en un recurso eficaz para la mejoría de la escritura de las estudiantes.

Cremades; Maqueda Cuenca y Onieva (2016) presentan el estudio de Gómes Camacho y Gómez del Castillo (2015) en la Universidad de Sevilla sobre la relación entre los errores ortográficos y la escritura usual en los mensajes de texto. El estudio fue desarrollado con 42 universitarios del curso de Maestro de Educación Infantil, que produjeron un texto académico y transcribieron mensajes propios enviados por WhatsApp. Como resultado se obtuvo que las dificultades ocasionales detectadas en algunos estudiantes también aparecían en sus mensajes en la aplicación móvil. Se concluyó, por lo tanto, que los problemas ortográficos de los hablantes residen en su formación en la lengua culta y no en la variedad lingüística utilizada. Es decir, los textismos de la aplicación no condicionan el desempeño en la escritura académica y la nueva norma utilizada por los hablantes cultos en los contextos multimedia está limitada apenas a esta situación comunicativa.

Estos estudios refuerzan que la utilización de recursos de las aplicaciones móviles puede resultar en disminución de la discriminación de que el lenguaje simplificado que se utiliza en estos contextos perjudica la práctica del lenguaje culto. En este sentido los profesores pueden pedir que los estudiantes utilicen una variedad lingüística más culta y sin abreviaciones para practicar la lengua meta en actividades fuera de clase.

Carvalho e Garrasini (2016) llevaron a cabo una investigación con sesenta alumnos del tercer año de la enseñanza media de una escuela pública en Brasil - GO, desarrollando actividades con la utilización del aplicativo de mensajería WhatsApp. El objetivo fue usar la tecnología en contexto híbrido y promover un aprendizaje significativo crítico y mayor tiempo de contacto con la lengua española. Analizando las interacciones en el aplicativo, las investigadoras constataron que ese ambiente les proporcionó a los alumnos: discutir y compartir informaciones sobre los temas abordados en la actividad, negociar y tomar decisiones de forma más rápida, aclarar dudas con la profesora del grupo y aprender de forma colaborativa. Por fin, el uso del aplicativo se reveló una excelente herramienta pedagógica para motivar y contribuir con una participación más activa de los estudiantes en su aprendizaje. 


\section{Metodología}

Para esta investigación elegimos una metodología cualitativa, la observación participante y el estudio de caso. De acuerdo con Richardson (2015, p. 90), la investigación cualitativa intenta "comprender en detalle los significados y las características situacionales presentadas por los encuestados en lugar de producir medidas cuantitativas de características o comportamientos".

Marconi y Lakatos (2017) afirman que este tipo de estudio se desarrolla en situaciones naturales y ofrece una gama de datos descriptivos con el foco en la realidad compleja y contextualizada. En este sentido, los autores atestan que el estudio de caso se identifica como una de las posibilidades de la investigación cualitativa, pues se refiere a la búsqueda más profundizada de informaciones sobre un caso o grupo en todos sus aspectos. Es el responsable por detallar la información o describir la complejidad de un hecho. Por fin, la observación participante es una de las técnicas más utilizadas en la investigación cualitativa para la recolección de datos. En ella, según Severino (2007), el investigador, para realizar el estudio de los fenómenos del grupo, comparte la experiencia de los sujetos investigados, participa de manera sistemática y permanente durante la explotación. También, integra las situaciones vividas y registra descriptivamente lo que se experimentó durante su interacción en las situaciones observadas.

Así para la recolección de los datos elegimos el cuestionario, la aplicación móvil de mensajería y la entrevista colectiva hecha con los grupos en sala. Es importante reforzar nuevamente que esta investigación fue realizada en 2019, o sea, antes del periodo pandémico vivenciado entre 2020 y el actual año de 2021. En los cuestionarios aclaramos las informaciones que ya teníamos sobre el perfil de los participantes y obtuvimos las respuestas a tres cuestiones: ¿Ya habías hecho algún trabajo en grupo con la utilización de la aplicación de mensajes? ¿Tuviste o no alguna dificultad en desarrollar la actividad con tus compañeros en la aplicación de mensajes, cuál(es)? ¿Qué te pareció la propuesta de trabajo que utiliza WhatsApp como herramienta pedagógica? A partir de las respuestas nuestro reto fue observar si en la opinión de los estudiantes la aplicación les ayudó a realizar la actividad y si la enseñanza híbrida fue efectiva para aumentar los espacios de aprendizaje y el tiempo de exposición a la lengua española.

En la aplicación buscamos percibir la interacción colaborativa de los alumnos para realizar la actividad y el proceso de desarrollo de la ZDP. En la entrevista, pretendemos confirmar las informaciones del cuestionario y obtener algunas otras pertinentes, que no hubieran sido expresadas en el cuestionario.

Los participantes fueron sesenta alumnos en total, con edades entre 13 y 16 años divididos en dos grupos, A y B con 30 alumnos en cada uno. La mayor parte de los alumnos estudia en la escuela desde la primaria, tres estudiantes ingresaron en el sexto año, una en el séptimo y dos en este año. Es decir, apenas tres estudiantes no empezaron los estudios de español en la escuela. Los alumnos de 
cada grupo fueron subdivididos en 6 grupos de cinco para poner en práctica una actividad. Lo propuesto fue que investigaran sobre cualquier tema de la cultura hispanoamericana para posterior presentación en un evento organizado por el departamento de lengua española que ocurre anualmente en la escuela investigada.

Para dar inicio a la propuesta tenían que elegir el tema de la investigación y decidir cómo les gustaría presentarlo, basados en las modalidades de presentación del evento (exposición, taller, ponencia oral y presentación artística). Los estudiantes deberían crear grupos en la aplicación móvil de mensajería WhatsApp para interactuar entre sí y con la profesora del grupo. Todas las decisiones y acciones para implementar la actividad deberían ser discutidas en la aplicación. Durante la explicación de la propuesta en clase la profesora les pidió que hablaran (grabación en audio) y escribieran siempre en español en la aplicación. Siempre que necesario podrían pedir aclaraciones o discutir algo con la profesora en clase.

\section{Selección y análisis de datos}

Para la descripción de los datos obtenidos se buscó a partir de la aplicación móvil de mensajería las conversas que expusieron la interacción colaborativa, el andamiaje y el desarrollo de la ZDP, también las respuestas al cuestionario y a la entrevista colectiva en el aula con los estudiantes que expresaron la opinión de la mayoría sobre la propuesta. Las transcripciones de las interacciones colaborativas son presentadas con los errores típicos de esta fase de aprendizaje. Ya las transcripciones de los trechos del cuestionario y de la entrevista fueron corregidas por las investigadoras pues no son relevantes para el entendimiento del discurso de los participantes.

Los datos aquí presentados son el resultado de una actividad desarrollada en los octavos años de la Enseñanza Fundamental, en una escuela pública Federal de la región norte de Goiânia - GO y fueron analizados a partir de los supuestos teóricos descritos anteriormente. Para representar a los participantes utilizaremos (PP) cuando se refiera a la profesora participante y (P1, P2, P3...), para los alumnos.

En los trechos a seguir presentamos las interacciones colaborativas entre los participantes de algunos grupos y, en algunos momentos, se percibe el desarrollo de la ZDP durante el proceso de colaboración.

\section{Trecho 1}

P1- "Ola, alguem sabe si es para escrever só en español aqui" [sic] 
P2 -"Hola, la profe habló en clase que tenemos que tentar hablar só en español. Si no sabemos una palavra o estrutura podemos perguntar en el grupo o pesquisar en los materiales de la disciplina, diccionario ou internet" [sic] P1- "Valeu, e que não vim ontem y me explicaron mas yo tive dubidas. Pensei que sería mejor perguntar en el grupo caso ninguém subiesa la profe me responderia" [sic]

PP - "Hola, bienvenido al grupo su colega le explicó perfectamente fue exactamente eso lo que dije cuando expliqué la actividad y la utilización del grupo en la aplicación. Cualquier duda que tengan pregúntenme aquí, ¡Buen trabajo!"

\section{Trecho 2}

P3 - "Encontrei bastante informaciones en mi pesquisa sobre Shakira pero tiene unas partes del texto que yo no sei qué significan alguem pode me ajudar. Enviei la foto del texto con las partes en amarello." [sic]

P4 - "Sí, son conectores del texto, hoje leímos un texto en el libro que aparecía esas palavras "sin embargo", ¿no te recordas?" [sic]

P3 - "Non, provavelmente estava conversando con mi colegas" [sic]

P4 - "Bueno, son palavras que significa una oposición entre lo que fue ditho antes y o que es ditho después" [sic]

P3 - "Entoces, ela non completa el texto son ideas diferentes? Non entendí biem." [sic]

P4 -"Vamos a ver no texto: El álbum Magia fue elegido para representar Colombia en el festival...sin embargo no pudo participar porque no tenía la edad mínima. ¿Quál es la primeira idea?" [sic]

P3 - "Seu álbum... gañó para representar o país no festival" [sic]

P4 - "Y después qué pasó? [sic]

P3 - "Ela non foi porque no tiña edade" [sic]

P4 - "Entonces para juntar las dos ideas de gañar para ir no festival y después no poder ir porque no tener la edad quál es o significado da estrutura sin embargo?" [sic]

P3 - "Ela gañó mas non foi" [sic]

P4 - "Sí, como si fose mas, tambén dise la profe en la classe con todo, a pesar de que" [sic]

P3 - "Ah, entendí, muthas gracias, voi aprender bastante en esse grupo que tiem los melhores alunos da classe" [sic]

PP - "Muy buena explicación P4, igualito yo haría"

P4 - "Gracias profe, fiz o raciocinio como tú fizeste con nosotros na classe quando apareció no texto do libro" [sic]

\section{Trecho 3}

P5 - "Me gustaría saber que significa ha puesto que aparece no texto sobre nuesso plato. Busqué en mio diccionario pero só aparecen as palavras separadas y no pareceu tener sentido la frase" [sic]

PP - "Por favor ¿puedes poner el texto aquí para saber el contexto?

P5 - "Sim claro, voi poner la foto do texto." [sic]

PP - "P5, ha puesto es una estructura verbal en pasado es uno de los 4 pasados que hay en español, como no estudiamos aun no lo reconociste"

P5 - "Entonces eres pasado, mas no dicionario dice que ha eres el presente do verbo haber" [sic]

PP - "Sí, esta forma verbal es compuesta por el verbo auxiliar haber conjugado en presente y el participio del verbo principal"

P5 - "Si, foi lo que encontré en el diccionario que es lo participio do verbo poner, mas no consigo entender que significa" [sic]

PP - "Bueno, este verbo es una estructura que indica un pasado reciente algo que ocurrió en pasado hace poco tiempo o que puede tener relación con el presente, por eso es compuesto por el verbo auxiliar en presente."

P5 - "Entendí, mas significa que tiene puesto algo" [sic]

PP - "No, no da la idea de acción continuada en pasado, significa que puso solo que fue recientemente, hace poco tiempo o fue en un tiempo que puede tener relación con el presente. En el texto aparece "se ha puesto el horno para calentar diez minutos antes" ¿cómo lo entiendes después de la explicación?

P5 - "Entonces, puso el horno para calentar, só que foi face 10 minutos" [sic]

PP - Es eso.

P5 - "Entendí, mas profe por que no dissen puso apenas? [sic]

PP - "Vea, es una forma verbal muy usual en España, en América no se utiliza, se usa puso, pero tenemos que aprender porque aparece en los textos."

P5 - "Si, gracias profe por la explicação, entendí y ja he puesto essa forma verbal nos mis apuntes aquí" [sic]

PP - "Veo que lo has entendido bien. Si hay más dudas es sólo preguntar" 
En la primera interacción (trecho 1) es posible percibir que un estudiante que no vino a la clase tiene dudas en cómo interactuar en el grupo. Uno de los integrantes le aclara sobre las orientaciones que la profesora dio en la clase. El participante habla que había tenido una orientación de otro colega fuera de la aplicación pero no estaba cierto de qué idioma utilizar y cómo proceder. La profesora refuerza la colaboración del colega cuando afirma que está correcta la orientación y deja abierta la oportunidad de aclaraciones siempre que necesario. En el trecho 2 observamos el andamiaje de la alumna P4 rehaciendo el camino de raciocinio que la profesora realizó en la clase cuándo explicó la misma estructura que apareció durante la lectura. En el trecho 3, el participante tiene dudas sobre una forma verbal todavía no estudiada en el $8^{\circ}$ año. La profesora participante le da un andamiaje sobre la forma y el uso de la estructura y al final (P5) hace uso de la estructura en una frase lo que señala que en ese momento ha internalizado la explicación. Los trechos refuerzan lo que afirma Hall (2001) sobre la interacción entre profesor y alumno que es la mejor herramienta para el aprendizaje. También los supuestos de Figueiredo (2019, p. 64) sobre el aprendizaje colaborativo y el papel de la interacción y de la colaboración en el trabajo entre pares o en grupos de estudiantes como una manera de fomentar la construcción del conocimiento. Percibimos que, está de acuerdo con Vygotsky (2000), cuando explica que la Zona de Desarrollo Próximo (ZDP) se refiere a las habilidades no adquiridas pero que pueden demostrarse y/o adquirirse por medio de la cooperación de alguien más experimentado.

Las respuestas a las preguntas del cuestionario dan la dimensión sobre lo que la actividad colaborativa significó para los estudiantes. En respuesta a la primera pregunta constatamos que la enseñanza híbrida y el uso de la aplicación móvil de mensajería son recursos poco trabajados en otras disciplinas de la EF.

\section{Trecho 4}

P19 - "Nunca había utilizado el WhatsApp para hacer un trabajo de la escuela."

\section{Trecho 5}

P23 - "Ya había utilizado el móvil para sacar fotos de plantas para la clase de biología, pero fue la primera vez que utilicé el WhatsApp para conversar con el grupo de un trabajo. Las decisiones salieron mucho más rápidas."

\section{Trecho 6}

P8 - "El año pasado utilizamos el celular una vez en la clase de informática porqué la profe trabajó con $Q R$ code y teníamos que usar una aplicación para leer los códigos. Pero nunca utilicé el celular para trabajar en conjunto con mis colegas. Lo bueno es que teníamos que escribir en español y eso fue bueno para mí."

\section{Trecho 7}

P4 - "En las clases de Arte utilizamos el celular para hacer nuestras propias interpretaciones de algunas obras de pintores famosos. Teníamos que buscar la pintura en el internet. Pero nunca hubo una propuesta de utilizar el WhatsApp para hacer un trabajo."

\section{Trecho 8}

P3 - "Nunca utilice el WhatsApp para hacer alguna actividad propuesta por el profesor. A veces converso con uno u otro colega por la aplicación pero nunca me ocurrió crear un grupo para conversar con los colegas cuando estamos 
haciendo un trabajo y lo mejor con la profe para ayudar a sacar las dudas o auxiliar cuando no sabemos si estamos haciendo lo correcto. Pero lo mejor era tener que escribir en español y aprender muchas palabras nuevas 0 estructuras que los colegas utilizaron, porque en mi grupo estaban los mejores de la clase y aprendí mucho."

En los trechos es posible percibir que los participantes ya realizaron otras actividades con el uso del móvil y de aplicaciones, pero nunca una propuesta de interacción colaborativa en lengua extranjera. Se percibe también que de manera autónoma los estudiantes no utilizan este recurso para otras actividades escolares lo que está de acuerdo con lo que explicó Souza (2015) sobre la utilización de las aplicaciones, el papel del profesor como mediador del proceso y del alumno de la generación digital que no posee autonomía para buscar la ampliación de sus capacidades de aprendizaje cuando el objetivo está impregnado del sentimiento de deber escolar. Aún, lo que afirman Horn y Staker (2015) que un abordaje híbrido bien implementado forma la base para un aprendizaje centrado en el alumno.

En el trecho 8, se percibe que los estudiantes no utilizan el recurso de crear grupos de discusiones en las disciplinas o para realizar actividades con los colegas.

La segunda pregunta aclaró alguna dificultad en la utilización de la aplicación como herramienta pedagógica. Se percibe en los trechos que la mayor parte de las dificultades está asociada a problemas técnicos y de relación familiar, apenas tres estudiantes demostraron dificultades con la escritura en lengua española.

\begin{abstract}
Trecho 9
P9-"Yo vivo en una ciudad cerca de Goiânia y ahí el internet es por medio del radio, entonces en algunos momentos no recibía los mensajes de mis colegas de grupo. Cuándo recibía ellos ya habían discutido muchas cosas y yo me quedaba perdido."
\end{abstract}

\title{
Trecho 10
}

P35 - "Como tengo solo trece años mi mamá no me deja estar todo el tiempo con el celular entonces, al principio solo podía estar una hora conversando con mis colegas. Como fue mi padre que firmó el papel para participar de la investigación le enseñé para que me dejara estar más tiempo. Aun así ella estuvo desconfiada y después que la profe le mandó un mensaje explicando la actividad nuevamente y cuándo iba a terminar ella me dejó ver los mensajes a cualquier momento. Gracias profe.

\section{Trecho 11}

P48 - "Yo soy evangélica y no tengo celular, mi mamá me prestó el suyo para hacer el trabajo, pero sólo estaba con él por la tarde, eso dificultó un poco porque ya había muchos mensajes para leer. Me gustó mucho trabajar en WhatsApp, escribir en español. Me gustaría tener un celular como mis colegas, creo que sería más fácil."

En los trechos percibimos que los problemas mencionados fueron relacionados a la velocidad del internet, la organización de la familia cuando al uso de los electrónicos y del internet y la religión que influye en la adquisición del aparato móvil. Los otros participantes de los dos grupos se limitaron a contestar "no" para esta pregunta, apenas estos tres participantes mencionaron algún tipo de dificultad que no tuvo relación con dificultades para trabajar con la aplicación. Realmente, PP tuvo que mantener 
contacto por la aplicación con una de las madres que desconfió de la necesidad de contestar a los mensajes. La entendimos pues hay riesgos de conversaciones maliciosas en la aplicación como en cualquier otro mecanismo social en red. Las respuestas a la segunda cuestión confirman lo que afirma Padrón (2013) que WhatsApp hoy en día es la aplicación para móviles más usual entre las personas, por la facilidad de comunicación en tiempo real, por compartir fotos, música y videos gratuitamente, así se puede agregarla en el aula para la comunicación entre los alumnos y el profesor.

En las opiniones de los participantes la utilización de la aplicación de mensajería trajo beneficios para el aprendizaje del español y se convirtió en una ampliación de las posibilidades de uso de la lengua en otros contextos fuera de la clase.

\begin{abstract}
Trecho 12
P3 - "Me gustó mucho trabajar en el WhatsApp con los colegas y la profe. Ya hablé que nunca me ocurrió crear grupos para trabajar con los colegas en los trabajos de las disciplinas. Lo más importante fue escribir en español, yo aprendí muchas palabras y estructuras con mis colegas (los mejores de la clase) y con la profe. También me pareció bueno que ella siempre aclaraba las dudas que teníamos. La actividad fue hecha más rápidamente, porque no teníamos que esperar la próxima clase para preguntar algo, ya teníamos la respuesta en el mismo día."
\end{abstract}

\title{
Trecho 13
}

P4 - "Me encantó utilizar el WhatsApp ya combinamos que vamos a sugerir a los otros profesores porque facilita mucho el trabajo. Pesquisé mucho para nuestra presentación, lo mejor fue que pudimos elegir lo que queríamos presentar y me encantó pesquisar sobre Shakira, no sabía que ella ayudaba a tantos niños en Colombia, fue bueno para conocer mejor la cantante. Me gustó mucho cuando terminamos la presentación y se la enviamos a la profe en la aplicación y ella ya contestó pidiendo que corrigiéramos muchas cosas y dio consejos para la presentación"

\section{Trecho 14}

P47 - "Yo creo que utilizar el WhatsApp fue una idea muy buena de la profe, pues estamos siempre con el móvil en la mano y nos gusta tanto utilizarlo que yo ni percibí que estaba haciendo un trabajo de español. Fue importante para hacer más rápido porque no teníamos que esperar al otro día para conversar en el recreo con los colegas porqué algunos del grupo yo no tengo el número y una colega no tiene móvil. Pero para hacer el trabajo utilizó el aparato de su madre y podíamos conversar con todos del grupo por la tarde.

Los trechos presentados traducen bien lo que la mayor parte de los participantes contestó, de que fue buena la experiencia por la rapidez de la interacción con los colegas y por poder sacar dudas con la profesora. Otras informaciones pertinentes como aprender estructuras en español e investigar sobre un tema de su interés están evidenciados también en los trechos. Otro dato importante fue la de hacer sugerencia a los profesores de otras asignaturas para que utilicen el recurso, pues les pareció efectivo y no habían tenido esa idea antes. Estas respuestas están en acuerdo con el resultado del estudio de Alsaleem (2013) mencionada por Cremades; Maqueda Cuenca y Onieva (2016), de que el uso del recurso electrónico se convirtió en un una herramienta eficaz para la mejoría de la escrita de los estudiantes en una LE. Se percibe en las respuestas de los participantes que desde que se tenga un aparato móvil y haya conexión con internet se puede practicar la lengua en actividades extra clase, como afirma Souza (2015). 
Durante las entrevistas colectivas con los grupos, los estudiantes reforzaron lo que habían dicho sobre la utilización de la aplicación y evidenciaron nuevos aspectos que no habíamos pensado para esta actividad. Como sugerencias los participantes describieron muchas otras posibilidades de utilización de la aplicación en clases de español y en otras asignaturas.

\title{
Trecho 15
}

P10 - "Yo pienso que este tipo de actividad que utiliza la aplicación WhatsApp puede ser utilizada en todos los trabajos en grupo. Me pareció muy bueno para aprender palabras nuevas y estructuras con la profe. Pienso que podíamos crear un grupo grande con toda la clase para poner ahí algunas cosas que encontramos interesante sobre la lengua, o poner videos de los alumnos hablando la prueba oral o cualquier cosa para corregir la pronunciación."

\begin{abstract}
Trecho 16
P55 - "Profe, mi madre es muy desconfiada a todo el momento venía a mi habitación a ver qué estaba pesquisando en el internet y con quienes estaba conversando en el grupo de WhatsApp, por fin se calmó y me dijo que nunca me había visto haciendo un trabajo tan compenetrado y sin reclamar. Realmente, me gustó mucho esta actividad pesquisé un tema que elegimos en el grupo, las cosas salieron rápido, las dudas eran solucionadas en el mismo día. Quiero siempre hacer actividades como esta. En otra oportunidad me gustaría hacer videos con mis colegas como una actividad que simula un telediario con entrevistas y reportajes con personajes que parezcan personas de la calle."
\end{abstract}

\section{Trecho 17}

P23 - Me gustó mucho la actividad en la aplicación WhatsApp porque fue bueno utilizar la aplicación que utilizamos todos los días en la tarea de español ella es muy buena porque podemos fotografiar algo y enviar para los colegas y ellos contestan rápidamente. Sacar las dudas contigo leer la explicación que nos dio de algunas cosas de la lengua española como si estuviéramos aprendiendo en una clase virtual. Me encanta las lenguas extranjeras y sobretodo el español, cuando fuera profe voy a hacer siempre esas actividades distintas con los alumnos como tú hiciste con nosotros. Porque quiero ser profe o de lengua extranjera o de biología que me encanta también.

Todos los participantes encontraron efectiva la actividad que utilizó la aplicación móvil de mensajería como herramienta pedagógica. Estos trechos evidenciaron algunos aspectos importantes en la propuesta. Percibimos que la aplicación sirvió para el aprendizaje de nuevas palabras y estructuras de la lengua. También estuvo evidente que fue una actividad motivadora incluso en la percepción de la madre de uno de los participantes. Y, por fin, la impresión de la participante de que la aplicación funcionó como una clase virtual lo que atestigua que la enseñanza híbrida puede convertirse en una posibilidad más de aprendizaje para los estudiantes. En las palabras de Moran (2015) que hoy día, a causa de la movilidad y conectividad, se puede enseñar y aprender de distintas formas, en todos los momentos y en múltiples espacios, confirman las palabras de la participante. También lo que afirman Basich; Tanzi Neto y Trevisani (2015) cuando explican que dentro de la perspectiva de educación híbrida el medio online da autonomía y flexibilidad al estudiante, además de permitirle que aprenda solo o en interacción con otros y explote sus capacidades por medio de la colaboración siempre que sea posible fuera del aula.

Constatamos, por lo tanto, que la propuesta de enseñanza híbrida con la utilización de la aplicación móvil de mensajería como herramienta para aumentar el tiempo de exposición de los aprendices y promover la interacción colaborativa fue productiva, motivadora y participativa en la visión 
de los participantes. La interacción ubicua fue algo que les llamó la atención y el intercambio de ayudas y el andamiaje fue percibido en las interacciones colaborativas como afirman los autores. Muchas otras ideas de utilización en las clases de español fueron listadas por los alumnos y con sugerencia de uso para otras asignaturas. Además de los estudiantes, la familia también percibió la motivación para la realización de las actividades y aunque no fue mencionada la enseñanza híbrida, una participante percibió la herramienta como una clase virtual.

\section{Consideraciones}

Este estudio evidenció todo el potencial de la utilización de las aplicaciones móviles como herramientas para el desarrollo de la LE en la escuela pública. Aunque sea poco el tiempo en clase con los alumnos hay posibilidades de multiplicar los espacios y el contacto con la lengua por medio de la tecnología.

Además, a partir de la educación híbrida es posible desarrollar más la capacidad de autonomía en el aprendizaje, pues el estudiante se vuelve un investigador que busca la solución de sus dudas o algo que desee aprender por sí mismo. Esta característica es excelente no solo para los estudios como también para la vida, ya que incentiva al aprendiente a hacerse responsable por su aprendizaje y su desarrollo cognitivo.

Con la utilización de la aplicación fue posible observar la interacción de los estudiantes entre sí y con la profesora, el intercambio de ayuda y el uso de la ZDP. Las respuestas al cuestionario evidenciaron que los estudiantes encontraron la propuesta efectiva y práctica. Les pareció interesante complementar las discusiones en la aplicación ya que el recreo en la escuela no se confirmó como un tiempo suficiente para las interacciones y negociaciones del grupo en otras ocasiones. Otro aspecto presentado en el estudio fue la presencia de la profesora en el ambiente virtual lo que les dio a los alumnos seguridad en la planificación y confirmación que estaban haciendo el trabajo de la manera correcta.

\section{Referencias}

ALSALEEM, B. I. A. The efects of WhatsApp electronic dialogue journaling on improving writing vocabulary word choice and voice of EFL undergraduate Saudi students. In: AWEJ- Arabian World English Journal. volume 4 número 3. 2013. P. 213-225. Disponible en: <<https://www.awej.org/images/Alllssues/Volume4/Volume4Num ber3Sept2013/18.pdf>>. Acceso en 19 de julio de 2019. 
BACICH, L.; TANZI NETO, A.; TREVISANI, F. M. (Orgs). Ensino Híbrido: personalização e tecnologia na educação. Porto Alegre/RS: Penso, 2015. p. 27-45.

CARVALHO, I. O. A prática colaborativa de leitura em língua espanhola: análise dos processos interativos de interpretação textual e resolução de dificuldades inferenciais. 2003. dissertação de Mestrado - Faculdade de Letras, Universidade Federal de Goiás, Goiânia, 2003.

CARVALHO, I. O.; GARRASINI, R. B. S. Atividade Colaborativa Significativa: uso do whatsapp nas aulas de língua espanhola. In. Veredas Escolares II. Partilhando experiências criativas de ensino e aprendizagem do CEPAE/UFG. (Orgs.) SUANNO, M. V. R.; SILVA, R. L. B. R.; FLEURY, V. F. Goiânia - Editora Espaço Acadêmico, 2016, p. 203-215.

CREMADES, R.; MAQUEDA CUENCA, E.; ONIEVA, J. L. Posibilidades didácticas de la escritura digital ubicua en la aplicación WhatsApp Messenger. In: Revista Letral. número 16. Granada/España: EUG/Editorial Universidad de Granada, 2016. p. 106-120. Disponible en: <<http://revistaseug.ugr.es/index.php/letral/article/view/4932 >>. Acceso en 18 de julio de 2019.

FIGUEIREDO, F. J. Q de. Correção com os pares: os efeitos do processo da correção dialogada na aprendizagem da escrita em língua inglesa. 2001. Tese (Doutorado em Letras) - Faculdade de Letras, Universidade Federal de Minas Gerais, Belo Horizonte. 2001.

A aprendizagem colaborativa de línguas: considerações conceituais e aplicações em distintos contextos. In: FIGUEIREDO, F. J. Q. de. (Org.). A aprendizagem colaborativa de línguas. 2 ed. Goiânia: Ed. da UFG, 2018, p. 13-57.

Vygotsky: a interação no ensino/aprendizagem de línguas. São Paulo: Parábola, 2019.

GÓMEZ CAMACHO, A.; GÓMEZ del CASTILLO, M. T. Escritura ortográfica y mensajes de texto en estudiantes universitarios. In: Perfiles Educativos. volumen 37, número 150. Ciudad Universitaria/México: UNAM, 2015. p. 91-104. Disponible en: « https://www.iisue.unam.mx/perfiles/articulo/2015-150escritura-ortografica-y-mensajes -de-texto-en-estudiantes-universitarios.pdf $>>$. Acceso en 19 de julio de 2019.

HALL, J. K. Classroom interaction and language learning. Ilha do Desterro. Florianópolis, n. 41, p. 17-39, 2001.

HORN, M. B.; STAKER, H. Blended: usando a inovação disruptiva para aprimorar a educação. Tradução de Maria Cristina Gularte Monteiro. Porto Alegre/RS: Penso, 2015.

MARCONI, M. A.; LAKATOS, E. M. Metodologia Científica. 7 ed. São Paulo: Atlas, 2017.

MORAN, J. Educação híbrida: um conceito-chave para a educação hoje. In: BACICH, L.; TANZI NETO, A.; TREVISANI, F. M. (Orgs). Ensino Hibrido: personalização e tecnologia na educação. Porto Alegre/RS: Penso, 2015. p. 27-45.

PADRÓN, C. J. Estrategias didácticas basadas en aplicaciones de mensajería instantánea WhatsApp exclusivamente para móviles (mobile learning) y el uso de la herramienta para promover aprendizaje 
colaborativo. In. EDUWEB Revista de Tecnología de Información y Comunicación en Educación. volumen 7, número 2. Vargas/ Venezuela: Universidad Simón Bolívar, 2013. p. 123-134. Disponible en: «< http://servicio.bc.uc.edu.ve/ educacion/eduweb/v7n2/art09.pdf >>. Acceso en 18 de julio de 2019.

PLESTER, B. WOOD, C. Exploring relashioships between traditional and new media literacies: british preteen texters at school. In: Journal of Computer-Mediated Communication. Número 14, 2009. p. 11081129. Disponible en: << https://academic.oup.com/jcmc/article/14/4/1108/4583569 > . Acceso en 19 de julio de 2019.

REGO, T.C. Vygotsky: uma perspectiva histórico-cultural da educação. $12^{a}$ ed. Rio de Janeiro: Vozes, 2001.

RICHARDSON, R. J. et al. Pesquisa Social: métodos e técnicas. 3 ed. São Paulo: Atlas, 2015.

SEVERINO, A. J. Metodologia do trabalho científico. 23 ed. São Paulo: Cortez, 2007.

SOUZA, C. F. Aprendizagem sem distância: tecnologia digital móvel no ensino de Língua Inglesa. In: Texto Livre-Linguagem e Tecnologia, ano 1, volume 8. Belo Horizonte/MG: UFMG, 2015.

VYGOTSKY, L. S. A formação social da mente. 6 ed. São Paulo: Martins Fontes, 2000.

WOOD, D.; BRUNER, J. S. and ROSS, G. The role of Tutoring in Problem Solving. Journal of Child Psychology and Psychiatry, v. 17, p. 89-100, 1976.

Data de submissão: 13/09/2021. Data de aprovação: 28/10/2021. 